\title{
Characterizing and prognosticating chronic lymphocytic leukemia in the elderly: prospective evaluation on 455 patients treated in the United States
}

Chadi Nabhan ${ }^{1 *}$, Anthony Mato ${ }^{2}$, Christopher R. Flowers ${ }^{3}$, David L. Grinblatt ${ }^{4}$, Nicole Lamanna ${ }^{5}$, Mark A. Weiss ${ }^{6}$, Matthew S. Davids ${ }^{7}$, Arlene S. Swern ${ }^{8}$, Shriya Bhushan ${ }^{8}$, Kristen Sullivan $^{9}$, E. Dawn Flick ${ }^{10}$, Pavel Kiselev ${ }^{8}$ and Jeff P. Sharman ${ }^{11}$

\begin{abstract}
Background: Median age at diagnosis of patients with chronic lymphocytic leukemia (CLL) is $>70$ years. However, the majority of clinical trials do not reflect the demographics of CLL patients treated in the community. We examined treatment patterns, outcomes, and disease-related mortality in patients $\geq 75$ years with CLL (E-CLL) in a real-world setting.

Methods: The Connect ${ }^{\oplus} \mathrm{CLL}$ registry is a multicenter, prospective observational cohort study, which enrolled 1494 adult patients between 2010-2014, at 199 US sites. Patients with CLL were enrolled within 2 months of initiating first line of therapy (LOT1) or a subsequent LOT (LOT $\geq 2$ ). Kaplan-Meier methods were used to evaluate overall survival. CLL- and infection-related mortality were assessed using cumulative incidence functions (CIF) and cause-specific hazards. Logistic regression was used to develop a classification model.
\end{abstract}

Results: A total of 455 E-CLL patients were enrolled; 259 were enrolled in LOT1 and 196 in LOT $\geq 2$. E-CLL patients were more likely to receive rituximab monotherapy $(19.3 \mathrm{vs.} 8.6 \% ; p<0.0001)$ and chemotherapy-alone regimens $(p<0.0001)$ than younger patients. Overall and complete responses were lower in E-CLL patients than younger patients when given similar regimens. With a median follow-up of 3 years, CLL-related deaths were higher in E-CLL patients than younger patients in LOT1 (12.6 vs. $5.1 \% p=0.0005)$ and LOT $\geq 2$ (31.3 vs. $21.5 \% ; p=0.0277)$. Infection-related deaths were also higher in E-CLL patients than younger patients in LOT1 (7.4 vs. 2.7\%; $p=0.0033$ ) and in LOT $\geq 2$ (16.2 vs. $11.2 \% ; p=0.0786$ ). A prognostic score for E-CLL patients was developed: time from diagnosis to treatment $<3$ months, enrollment therapy other than bendamustine/rituximab, and anemia, identified patients at higher risk of inferior survival. Furthermore, higher-risk patients experienced an increased risk of CLL- or infection-related death (30.6 vs 10.3\%; $p=0.0006)$.

Conclusion: $C L L$ - and infection-related mortality are higher in CLL patients aged $\geq 75$ years than younger patients, underscoring the urgent need for alternative treatment strategies for these understudied patients.

Trial Registration: The Connect CLL registry was registered at clinicaltrials.gov: NCT01081015 on March 4, 2010.

Keywords: Chronic lymphocytic leukemia, Connect ${ }^{\oplus}$ CLL registry, Elderly, Prognostic, Chemoimmunotherapy

\footnotetext{
* Correspondence: chadi.nabhan@cardinalhealth.com

${ }^{1}$ Cardinal Health Specialty Solutions, Waukegan, IL 60085, USA

Full list of author information is available at the end of the article
} 


\section{Background}

Chronic lymphocytic leukemia (CLL) accounts for 15000 diagnosed cases in the USA annually [1]. While incremental improvements in treating CLL have been observed in the past decade [2], the majority of clinical trials leading to these treatment approaches have largely enrolled younger, fitter patients who do not accurately reflect the demographics of CLL patients seen in the community [3-6]. One exception was the CLL-11 study that compared chlorambucil alone to chlorambucil combined with rituximab or obinutuzumab in patients with co-morbidities defined as either a glomerular-filtration rate $<70 \mathrm{~mL} / \mathrm{min}$ or a cumulative-illness-rating scale $\geq 6$ [7]. Other studies have allowed enrollment of elderly patients and performed unplanned subset analyses in an attempt to refine treatments and outcomes in the elderly, but data were inconclusive [8-10]. Moreover, a populationbased analysis of 28590 US patients diagnosed with CLL (1992-2009) showed that the improvement in overall survival (OS) noted in younger patients was less pronounced in the elderly [11]. Furthermore, Brenner et al. [12] showed that improved survival for CLL has not been observed in older patients.

Whether these differences are related to disparities in therapeutic choice, access to care, non-CLL-related deaths in elderly patients, or variations in CLL biology and prognostic indicators is unknown. As the median age of CLL patients at diagnosis approaches 72 years, understanding the biology and outcomes for elderly patients is critical and underscored by the reported inferior survival of these patients.

To examine treatment patterns and disease-related outcomes in elderly CLL patients (defined as $\geq 75$ years), we used the Connect $^{\circ}$ CLL database that enrolled 1494 CLL patients requiring therapy between 2010 and 2014 [13]. These patients were almost entirely enrolled prior to the introduction of novel B-cell receptor (BCR)-targeted therapies. We aimed to establish a benchmark for outcomes in elderly CLL patients treated before the availability of BCR-targeted therapies to help in properly positioning newer agents in the elderly CLL treatment paradigm. Our objective was to compare patient and disease characteristics, prognostic indicators, complications, and disease-related mortality. Further, we aimed to develop a prognostic score that predicts elderly CLL patients at highest risk of CLL- or infection-related deaths. To our knowledge, this represents the largest comprehensive, prospective evaluation of this patient population published to date.

\section{Methods}

\section{Study design and participants}

The Connect CLL registry (NCT01081015), a multicenter, prospective, observational cohort study enrolled 1494 CLL patients treated at 199 US community- and academicbased sites from March 2010 to January 2014 [13]. The study protocol was approved by a central institutional review board (IRB) (Quorum Review IRB, Seattle, WA, USA) or each site's IRB (Additional file 1). Eligible patients were $\geq 18$ years and had CLL as defined by the International Workshop on Chronic Lymphocytic Leukemia (IWCLL) guidelines [14]. Eligible patients were those initiating a first or higher line of therapy (LOT) within 2 months prior to study enrollment. Personnel were educated to enroll patients consecutively as they entered a LOT and to invite every eligible patient to participate in the registry. For this analysis, patients were divided into two groups based on LOT: first line of therapy (LOT1) and second line of therapy or greater (LOT $\geq 2$ ). Each patient was followed up for up to 60 months or until early discontinuation (i.e. due to death, withdrawal of consent, loss to follow-up, or study termination). Follow-up data were collected approximately every 3 months during study participation. Reasons for treatment initiation and responses were assessed by the treating physician.

\section{Statistical analysis}

Date of enrollment was considered baseline for this study. Only laboratory samples collected $<7$ days before the start of enrollment therapy were used for baseline laboratory testing. Disease and patients' characteristics, practice patterns, clinical outcomes, and disease-related mortality were assessed. Continuous variables were reported using measures of dispersion and central tendency (means, medians, ranges, and standard deviation [SD]); categorical variables were reported as numbers and percentages (proportionality, 95\% confidence intervals [CI]) of the total study population. Medical history at enrollment and pre-existing condition data were used to generate a Charlson Comorbidity Index (CCI) $[15,16]$. Results were summarized by LOT at enrollment (LOT1 or LOT $\geq 2$ ) and by age group ( $<75$ years and $\geq 75$ years). The Chi-square test for the comparison of rates was used to assess differences between patient subgroups. Statistical significance was assessed at $p=0.05$ (two-sided). The Breslow-Day test was used to assess the homogeneity of the odds ratios.

The Kaplan-Meier method was used to estimate survival, calculated from the date on which therapy was initiated [17]. $p$ value was derived from log-rank tests for comparison of survival distributions. CLL-related deaths due to disease progression were distinguished from deaths due to other causes and recorded by the treating physician. CLLor infection-related survival was assessed using cumulative incidence functions (CIFs); $p$ values from Gray's test for equality of CIFs were reported. Cause-specific hazards analysis identified predictors of survival in univariate and multivariable settings. Predictors demonstrating an 
association with time to event $(p<0.1)$ were included in multivariable analyses to identify significant independent predictors. Cause-specific hazard ratios (HR) and $95 \% \mathrm{CI}$ were calculated.

Predictive modeling using logistic regression and a $k$-fold cross-validation method with $k=5$ was used to develop a prognostic score for elderly CLL patients [18]. Results were confirmed by assessment of the interaction between the above covariates and the elderly CLL group in the analysis of all eligible patients. Statistical analyses were performed using SAS ${ }^{\circ}$ (version 9.2) statistical software (SAS Institute, Cary, NC, USA).

\section{Results}

\section{Patient characteristics}

Table 1 shows that of 1494 patients enrolled in the registry, 455 patients were $\geq 75$ years; 259 patients $\geq 75$ years were enrolled in LOT1 and 196 in LOT $\geq 2$. Patient demographics and disease characteristics were largely similar between patients enrolled in LOT1 and LOT $\geq 2$, with the exception of duration of CLL from diagnosis to enrollment (1.8 vs 7.2 years at $\mathrm{LOT} \geq 2$ ). Differences were also observed between patients aged $<75$ and $\geq 75$ years for Rai staging, constitutional symptoms, and ECOG score at LOT1, and for sex, time from diagnosis to first LOT, race, geographical region, and ECOG score at LOT $\geq 2$ (Table 1).

\section{Treatment patterns}

Elderly CLL patients were more likely to receive rituximab monotherapy than younger patients, regardless of LOT (19.3 vs. $8.6 \%$ in LOT $1 ; 15.3$ vs. $12.7 \%$ in $\mathrm{LOT} \geq 2$ ). This was significant for patients receiving LOT1 $(p<0.0001)$ (Table 2). Patients $\geq 75$ years in LOT $\geq 2$ were significantly less likely to receive bendamustine/rituximab (BR) than patients $<75$ years $(21.9$ vs. $30.6 \% ; p=0.0267)$. Only $6.9 \%$ of patients $\geq 75$ years in LOT1 received fludarabine/cyclophosphamide/rituximab (FCR), versus 33.7\% of patients $<75$ years $(p<0.0001)$. Interestingly, patients $\geq 75$ years were significantly more likely to receive chemotherapy alone without anti-CD20 antibody therapy than patients $<75$ years. This was true for LOT1 (20.1 vs. 10.3\%; $p<0.0001)$ and LOT $\geq 2$ (25.5 vs. $11.0 \% ; p<0.0001)$.

Geographic variations in treatment patterns were also observed. In elderly CLL patients in LOT1, the South had the highest utilization of rituximab-based regimens $(61.2 \%)$ while the West had the lowest $(29.2 \% ; p<0.0023)$. For patients covered by private insurance, younger CLL patients were more likely to receive rituximab-based therapies than elderly CLL patients (80.1 vs. $50.0 \% ; p<0.0001$ ). This was also observed for patients covered by other insurance providers including Medicare, Medicaid, and military health insurance ( 71.8 vs. $54.5 \% ; p<0.0001)$. When analyzed using the Breslow-Day test, the results did not differ significantly by health insurance coverage $(p=0.0879)$.

\section{Response and survival}

For all patients enrolled in LOT1, overall response rate (ORR) was $60.2 \%$ (38.1\% complete response [CR]) while patients enrolled in LOT $\geq 2$ had an ORR of $42.6 \%$ (17.0\% CR). In LOT1, ORRs were significantly lower in patients $\geq 75$ years compared with patients $<75$ years (ORR: 48.3 vs. $65.1 \%$ respectively; $p<0.0001$ and CR: 25.9 vs. $42.3 \%$, respectively; $p<0.0001)$. Lower ORR and CR were also observed for elderly CLL patients in LOT1 when specific enrollment therapies were analyzed (Additional file 2: Table S1). Similarly, lower ORRs were observed in $\mathrm{LOT} \geq 2$ (CR: 11.2 vs. $19.8 \% ; p=0.009$ ). As responses were investigator-assessed, we investigated whether patients were evaluated by imaging at enrollment. Patients $\geq 75$ years were less likely to be evaluated by imaging than patients $<75$ years $(65.4$ vs. $72.0 \%$; $p=0.004)$. This finding was maintained after adjusting for LOT.

\section{Outcomes}

As of August 25, 2015, with a median follow-up of 32.6 months for all 1494 patients, 433 (29\%) had died; causes of death are summarized in Fig. 1. As expected, OS was significantly lower in patients $\geq 75$ years than patients $<75$ years in both LOT1 (log-rank $p<0.0001$; Fig. 2a) and LOT $\geq 2$ (log-rank $p<0.0001$; Fig. 2b).

Notably, elderly CLL patients were more likely to die from CLL in LOT1 (12.6 vs. $5.1 \%$, Gray's test $p=0.0005$; Fig. 3a) and LOT $\geq 2$ (31.3 vs. $21.5 \%$, Gray's test $p=0.0277$; Fig. 3b). Time to death from CLL or infection in patients in LOT1 was also significantly shorter in patients $\geq$ 75 years than patients $<75$ years (Gray's test $p<$ 0.0001; Fig. 3c), and in patients in LOT $\geq 2$ (Gray's test $p=0.0014$; Fig. 3d). Analysis of cause-specific hazards was performed to identify predictors of death from CLL in patients enrolled in LOT1. In univariate analyses, insurance status, anemia, del(17p) abnormality, and age $\geq 75$ years (Additional file 3: Table S2) were identified as significant factors. Multivariable analysis retained age $\geq 75$ years at enrollment (HR: 3.66, 95\% CI 1.92-7.00), and the presence of the del(17p) abnormality (by fluorescence in situ hybridization or cytogenetic testing) (HR: 2.63, 95\% CI 1.20-5.78) as independent predictors of a higher risk of death.

\section{Prognostic model for early death from CLL or infection in elderly CLL patients}

We performed prognostic modeling on 181 elderly CLL patients receiving LOT1 who were followed up for $\geq 2$ years. Modeling was carried out using the $k$-fold cross-validation method. Due to the limited sample size, a 5-fold crossvalidation approach was chosen. The sample of 181 patients was randomly partitioned into five validation subsets of approximately equal size. Five models were generated using 
Table 1 Demographics and characteristics of patients at enrollment to therapy

\begin{tabular}{|c|c|c|c|c|c|c|}
\hline \multirow{3}{*}{ Characteristics } & \multicolumn{2}{|c|}{ LOT1 $(n=889)$} & \multirow{3}{*}{$p$ value $e^{a, b}$} & \multicolumn{2}{|c|}{$\mathrm{LOT} \geq 2(n=605)$} & \multirow{3}{*}{$p$ value ${ }^{a, b}$} \\
\hline & $<75$ years & $\geq 75$ years & & $<75$ years & $\geq 75$ years & \\
\hline & $(n=630)$ & $(n=259)$ & & $(n=409)$ & $(n=196)$ & \\
\hline \multicolumn{7}{|l|}{ Age, years } \\
\hline Mean & 62.4 & 80.4 & & 63.9 & 80.8 & \\
\hline SD & 8.26 & 4.33 & & 7.67 & 4.37 & \\
\hline Median & 63.0 & 80.0 & & 65.0 & 80.0 & \\
\hline Range & $22-74$ & $75-99$ & & $34-74$ & $75-96$ & \\
\hline \multicolumn{7}{|l|}{ Sex, n (\%) } \\
\hline Male & 411 (65.2) & 155 (59.8) & 0.1288 & $281(68.7)$ & $106(54.1)$ & 0.0005 \\
\hline Female & 219 (34.8) & $104(40.2)$ & & $128(31.3)$ & $90(45.9)$ & \\
\hline \multicolumn{7}{|c|}{ Duration of CLL from diagnosis to registry enrollment, years } \\
\hline Median & 1.4 & 1.8 & 0.2912 & 7.0 & 7.2 & 0.7074 \\
\hline Range & $0-29$ & $0-32$ & & $0-32$ & $0-30$ & \\
\hline \multicolumn{7}{|l|}{ Time from diagnosis to first LOT, years } \\
\hline Median & 1.4 & 1.8 & 0.2593 & 1.4 & 2.3 & 0.0139 \\
\hline Range & $0-29$ & $0-32$ & & $0-32$ & $0-20$ & \\
\hline \multicolumn{7}{|l|}{ Race, $n(\%)^{c, d}$} \\
\hline White & $561(92.3)$ & $237(92.9)$ & 0.7211 & $352(90.0)$ & $183(96.3)$ & 0.0076 \\
\hline Black & $40(6.6)$ & $16(6.3)$ & & $37(9.5)$ & $5(2.6)$ & \\
\hline American Indian/Alaskan native & 0 & 0 & & $1(0.3)$ & 0 & \\
\hline Asian & $3(0.5)$ & 0 & & $1(0.3)$ & 0 & \\
\hline Other & $4(0.7)$ & $2(0.8)$ & & 0 & $2(1.1)$ & \\
\hline \multicolumn{7}{|l|}{ Geographic region, n (\%) } \\
\hline Northeast & $75(12.0)$ & $37(14.3)$ & 0.2029 & $58(14.3)$ & $37(19.0)$ & 0.016 \\
\hline Midwest & 207 (33.2) & $70(27.1)$ & & $137(33.7)$ & $45(23.1)$ & \\
\hline South & $249(40.0)$ & $103(39.9)$ & & $162(39.8)$ & $77(39.5)$ & \\
\hline West & $92(14.8)$ & $48(18.6)$ & & $50(12.3)$ & $36(18.5)$ & \\
\hline \multicolumn{7}{|l|}{ Institution type, n (\%) } \\
\hline Academic & $74(11.7)$ & $12(4.6)$ & & $57(13.9)$ & $12(6.1)$ & \\
\hline Community & $545(86.5)$ & $242(93.4)$ & & $343(83.9)$ & $181(92.3)$ & \\
\hline Government & $11(1.7)$ & $5(1.9)$ & & $9(2.2)$ & $3(1.5)$ & \\
\hline \multicolumn{7}{|l|}{ Insurance, n (\%) } \\
\hline Medicare & $283(44.9)$ & $229(88.4)$ & & $227(55.5)$ & $175(89.3)$ & \\
\hline Medicaid & $28(4.4)$ & $14(5.4)$ & & $16(3.9)$ & 7 (3.6) & \\
\hline Supplemental coverage & $86(13.7)$ & $92(35.5)$ & & $81(19.8)$ & $67(34.2)$ & \\
\hline Private coverage & $357(56.7)$ & $46(17.8)$ & & 189 (46.2) & $35(17.9)$ & \\
\hline $\mathrm{HMO}$ & $88(14.0)$ & $16(6.2)$ & & $56(13.7)$ & $13(6.6)$ & \\
\hline PPO & $206(32.7)$ & $26(10.0)$ & & $103(25.2)$ & $14(7.1)$ & \\
\hline Other & $64(10.2)$ & $4(1.5)$ & & $33(8.1)$ & $8(4.1)$ & \\
\hline Military & $10(1.6)$ & $5(1.9)$ & & $5(1.2)$ & $6(3.1)$ & \\
\hline Self-pay & $13(2.1)$ & 0 & & $6(1.5)$ & 0 & \\
\hline Other Insurance & $10(1.6)$ & $3(1.2)$ & & $8(2.0)$ & $3(1.5)$ & \\
\hline Not specified & $15(2.4)$ & $5(1.9)$ & & $19(4.6)$ & $2(1.0)$ & \\
\hline
\end{tabular}


Table 1 Demographics and characteristics of patients at enrollment to therapy (Continued)

\begin{tabular}{|c|c|c|c|c|c|c|}
\hline \multicolumn{7}{|l|}{ ECOG score and status, $\mathrm{n}(\%)^{\mathrm{c}, \mathrm{d}}$} \\
\hline 0 - Fully active & $276(57.4)$ & $70(33.7)$ & $<0.001$ & $138(46.8)$ & $42(30.7)$ & 0.0105 \\
\hline 1 - Restricted in strenuous activity only & $180(37.4)$ & $116(55.8)$ & & $138(46.8)$ & $79(57.7)$ & \\
\hline 2 - Ambulatory, but unable to work & $22(4.6)$ & $19(9.1)$ & & $17(5.8)$ & $14(10.2)$ & \\
\hline 3 - Capable of only limited self-care & $2(0.4)$ & $3(1.4)$ & & $2(0.7)$ & $2(1.5)$ & \\
\hline 4 - Completely disabled & $1(0.2)$ & 0 & & 0 & 0 & \\
\hline \multicolumn{7}{|l|}{ Rai staging system score, $\mathrm{n}(\%)^{\mathrm{c}, \mathrm{d}}$} \\
\hline Stage 0 & $112(23.7)$ & $60(28.4)$ & 0.0219 & $63(25.2)$ & $46(32.6)$ & 0.1728 \\
\hline Stage I & $143(30.2)$ & $48(22.7)$ & & $58(23.2)$ & $39(27.7)$ & \\
\hline Stage II & $83(17.5)$ & $25(11.8)$ & & $43(17.2)$ & $16(11.3)$ & \\
\hline Stage III & $71(15.0)$ & $36(17.1)$ & & $44(17.6)$ & $24(17.0)$ & \\
\hline Stage IV & $64(13.5)$ & $42(19.9)$ & & $42(16.8)$ & $16(11.3)$ & \\
\hline Constitutional symptoms, n (\%) & $397(63.0)$ & $183(71.8)$ & 0.0192 & $264(65.0)$ & $126(64.3)$ & 0.977 \\
\hline Fatigue $e^{f}$ & $328(82.6)$ & $152(83.1)$ & & $212(80.3)$ & $114(90.5)$ & \\
\hline Fever & $44(11.1)$ & $16(8.7)$ & & $22(8.3)$ & $5(4.0)$ & \\
\hline Night sweats & $164(41.3)$ & $62(33.9)$ & & $85(32.2)$ & $27(21.4)$ & \\
\hline Other & $69(17.4)$ & $44(24.0)$ & & $54(20.5)$ & $25(19.8)$ & \\
\hline Weight loss & $97(24.4)$ & $60(32.8)$ & & $71(26.9)$ & $37(29.4)$ & \\
\hline \multicolumn{7}{|l|}{ Metaphase cytogenetic analysis, n (\%) } \\
\hline Yes & $254(40.3)$ & $93(35.9)$ & & $148(36.2)$ & $46(23.5)$ & \\
\hline Abnormalities found ${ }^{f}$ & $110(43.3)$ & $48(51.6)$ & & $81(54.7)$ & $23(50.0)$ & \\
\hline del(11q) & $24(9.4)$ & $12(12.9)$ & & $18(12.2)$ & $5(10.9)$ & \\
\hline $\operatorname{del}(13 q)$ & $36(14.2)$ & $11(11.8)$ & & $23(15.5)$ & $7(15.2)$ & \\
\hline Trisomy 12 & $41(16.1)$ & $16(17.2)$ & & $30(20.3)$ & $8(17.4)$ & \\
\hline del(17p) & $13(5.1)$ & $6(6.5)$ & & $12(8.1)$ & $3(6.5)$ & \\
\hline Other & $35(13.8)$ & $18(19.4)$ & & $36(24.3)$ & $10(21.7)$ & \\
\hline \multicolumn{7}{|l|}{ FISH analysis, $n(\%)^{e}$} \\
\hline Yes & $377(59.8)$ & $136(52.5)$ & & $157(38.4)$ & $67(34.2)$ & \\
\hline Abnormalities found ${ }^{f}$ & $281(74.5)$ & $99(72.8)$ & & $116(73.9)$ & $44(65.7)$ & \\
\hline del(11q) & $64(17.0)$ & $26(19.1)$ & & $31(19.7)$ & $14(20.9)$ & \\
\hline $\operatorname{del}(13 q)$ & $179(47.5)$ & $59(43.4)$ & & $69(43.9)$ & $30(44.8)$ & \\
\hline Trisomy 12 & 74 (19.6) & $31(22.8)$ & & $38(24.2)$ & $10(14.9)$ & \\
\hline $\operatorname{del}(17 p)$ & $33(8.8)$ & 18 (13.2) & & $28(17.8)$ & $9(13.4)$ & \\
\hline Other & $24(6.4)$ & $14(10.3)$ & & $11(7.0)$ & $6(9.0)$ & \\
\hline $\operatorname{ALC}\left(\times 10^{9} / L\right)$ & 263 & 106 & & 179 & 79 & \\
\hline Mean $\left(\times 10^{9} / L\right)$ & 68.5 & 58.4 & & 52.7 & 54.2 & \\
\hline SD & 75.7 & 62.9 & & 64.5 & 51.9 & \\
\hline Median $\left(\times 10^{9} / L\right)$ & 46.1 & 34.6 & & 25.6 & 40.1 & \\
\hline Range $\left(\times 10^{9} / \mathrm{L}\right)$ & $0-564.0$ & $1.3-275.4$ & & $0.1-306.0$ & $0.8-271.1$ & \\
\hline
\end{tabular}

${ }^{a} p$ values (bold text) calculated using a Chi-square test. ${ }^{b} p$ values of interest are shown. ${ }^{c}$ Data are missing. ${ }^{\mathrm{d}}$ Rounding of numbers may cause totals to be $=,<$, or $>$ $100 \%$. ${ }^{\mathrm{e}}$ More than one answer permitted. ${ }^{\mathrm{f} P e r c e n t a g e s}$ calculated based on the number of patients tested

ALC absolute lymphocyte count, CLL chronic lymphocytic leukemia, ECOG Eastern Cooperative Oncology Group, FISH fluorescence in-situ hybridization, HMO health maintenance organization, $L O T 1$ first line of therapy, $L O T \geq 2$ second line of therapy or greater, PPO preferred provider organization, SD standard deviation

this approach. In multivariable analyses, significant predictors of death due to CLL or infection included choice of enrollment therapy, CCI score, time from diagnosis, anemia at enrollment, sex, and race. However, validation of these models did not provide consistent results primarily due to the small size of the validation datasets. Therefore, a decision 
Table 2 Type of therapy by age group (most frequently used regimens)

\begin{tabular}{|c|c|c|c|c|c|c|}
\hline \multirow[t]{3}{*}{ Regimen } & \multicolumn{3}{|c|}{ LOT1 $(n=889)$} & \multicolumn{3}{|c|}{ LOT $\geq 2(n=605)$} \\
\hline & $<75$ years & $\geq 75$ years & $p$ value $e^{a, b}$ & $<75$ years & $\geq 75$ years & $p$ value $a, b$ \\
\hline & $(n=630)$ & $(n=259)$ & & $(n=409)$ & $(n=196)$ & \\
\hline Rituximab monotherapy, n (\%) & $54(8.6)$ & $50(19.3)$ & $<0.0001$ & $52(12.7)$ & $30(15.3)$ & 0.3834 \\
\hline Combinations with rituximab, n (\%) & $482(76.5)$ & $139(53.7)$ & $<0.0001$ & $243(59.4)$ & $90(45.92)$ & 0.0018 \\
\hline Bendamustine/rituximab & $126(20.0)$ & $61(23.6)$ & 0.2377 & $125(30.6)$ & $43(21.9)$ & 0.0267 \\
\hline Bendamustine/dexamethasone/rituximab & $5(0.8)$ & $4(1.5)$ & & $5(1.2)$ & 0 & \\
\hline Chlorambucil/rituximab & 0 & $4(1.5)$ & & 0 & 0 & \\
\hline Cyclophosphamide/rituximab & 0 & 0 & & 0 & $3(1.5)$ & \\
\hline Cyclophosphamide/fludarabine/dexamethasone/rituximab & $8(1.3)$ & 0 & & 0 & 0 & \\
\hline Cyclophosphamide/lenalidomide/rituximab & 0 & 0 & & $4(1.0)$ & 0 & \\
\hline Cyclophosphamide/pentostatin/rituximab & $21(3.3)$ & $3(1.2)$ & & $13(3.2)$ & 0 & \\
\hline Cyclophosphamide/vincristine/prednisone/rituximab & $14(2.2)$ & $9(3.5)$ & & $8(2.0)$ & $6(3.1)$ & \\
\hline Fludarabine/cyclophosphamide/rituximab & $212(33.7)$ & $18(6.9)$ & $<0.0001$ & $41(10.0)$ & $11(5.6)$ & 0.0700 \\
\hline Fludarabine/rituximab & $33(5.2)$ & $22(8.5)$ & & $14(3.4)$ & $11(5.6)$ & \\
\hline Lenalidomide/rituximab & $10(1.6)$ & 0 & & 0 & 0 & \\
\hline Prednisone/rituximab & 0 & $4(1.5)$ & & 0 & $3(1.5)$ & \\
\hline Investigational product/rituximab & $14(2.2)$ & 0 & & 0 & 0 & \\
\hline Chemotherapy alone, $\mathrm{n}(\%)$ & $65(10.3)$ & $52(20.1)$ & $<0.0001$ & $45(11.0)$ & $50(25.5)$ & $<0.0001$ \\
\hline Bendamustine & $23(3.7)$ & $8(3.1)$ & & $24(5.9)$ & $21(10.7)$ & \\
\hline Chlorambucil & $18(2.9)$ & $22(8.5)$ & & $6(1.5)$ & $12(6.1)$ & \\
\hline Chlorambucil/prednisone & 0 & $12(4.6)$ & & 0 & $3(1.5)$ & \\
\hline Cyclophosphamide & 0 & $4(1.5)$ & & 0 & 0 & \\
\hline Cyclophosphamide/fludarabine & $11(1.7)$ & 0 & & 0 & $2(1.0)$ & \\
\hline Cyclophosphamide/vincristine/prednisone & 0 & 0 & & $5(1.2)$ & 0 & \\
\hline Fludarabine & $9(1.4)$ & 0 & & $5(1.2)$ & $10(5.1)$ & \\
\hline Other, n (\%) & $14(2.2)$ & $16(6.2)$ & 0.0030 & $46(11.3)$ & $18(9.2)$ & 0.4400 \\
\hline Alemtuzumab & 0 & 0 & & $14(3.4)$ & $6(3.1)$ & \\
\hline Lenalidomide & 0 & $4(1.5)$ & & 0 & 0 & \\
\hline Ofatumumab & 0 & 0 & & $17(4.2)$ & $8(4.1)$ & \\
\hline Investigational product & $6(1.0)$ & $7(2.7)$ & & $13(3.2)$ & $4(2.0)$ & \\
\hline
\end{tabular}

${ }^{a} p$ values (bold text) calculated using a Chi-square test. ${ }^{b} p$ value shown for large patient groups only

$L O T 1$ first line of therapy, LOT $\geq 2$ second line of therapy or greater

was made to identify independent predictors of death among the covariates that were part of at least one multivariable model. These covariates were used in the final model.

Three predictors were identified: time from diagnosis to first treatment, enrollment therapy other than BR, and anemia. Based on the relative magnitude of effect, each predictor was weighted and assigned a score [19]. Time from diagnosis to treatment $<3$ months and therapy other than BR were assigned a score of 2; anemia at enrollment was assigned a score of 1 . Patients were classified into risk groups: lower-risk (score $\leq 4)$ and higher-risk (score $=5$ ). When stratified by risk, mortality due to CLL or infection was $10.3 \%$ in the lower-risk group $(n=145)$ compared with $30.6 \%$ in the higher-risk group $(n=36)$ (Chi-square $p=0.0002)$. This prognostic model was validated in a multivariate analysis of all patients with a grouping variable and interaction terms for each of the significant covariates.

\section{Serious adverse events}

Serious adverse events (SAEs) of any grade were more common in patients $\geq 75$ years than patients $<75$ years in LOT1 (56.0 vs. $39.4 \%)$ and LOT $\geq 2$ (68.4 vs. 61.9\%) (Additional file 4: Table S3). Grade $\geq 3$ SAEs were more common in elderly CLL patients in LOT1 (51.4 vs. 34.8\%) (Additional file 5: Table S4). The most frequent grade $\geq 3 \mathrm{SAE}$, pneumonia, was more common in elderly CLL patients in LOT1 $(9.7 \%)$ than in patients $<75$ years (4.0\%); however, in LOT $\geq 2$ rates of grade $\geq 3$ pneumonia 
a $n=630$

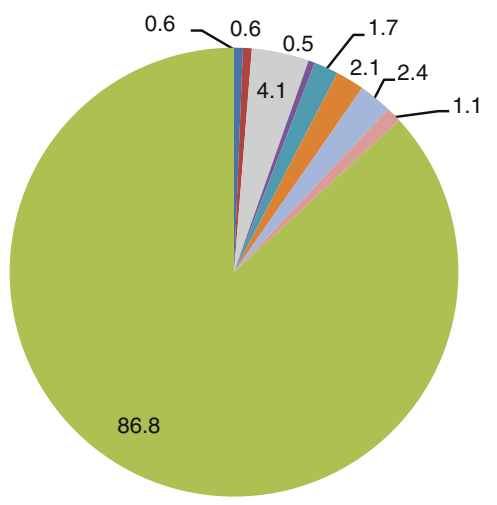

C $n=409$

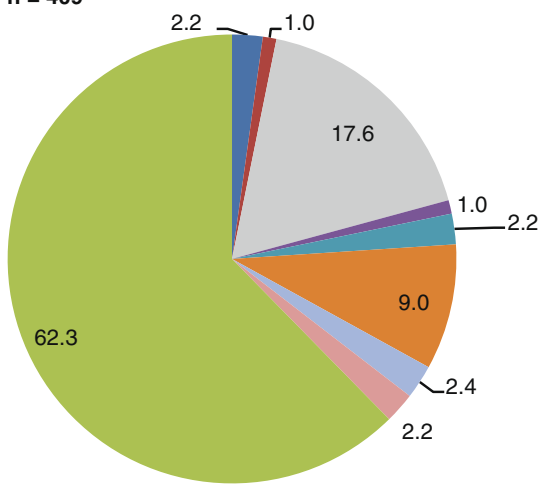

- Vardiac event
- Richter's transformation (Large B-cell lymphoma)

- Second primary malignancy

- Infection b $\quad n=259$

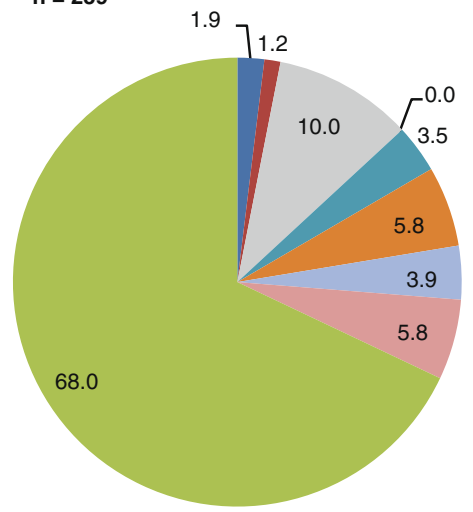

d $n=196$

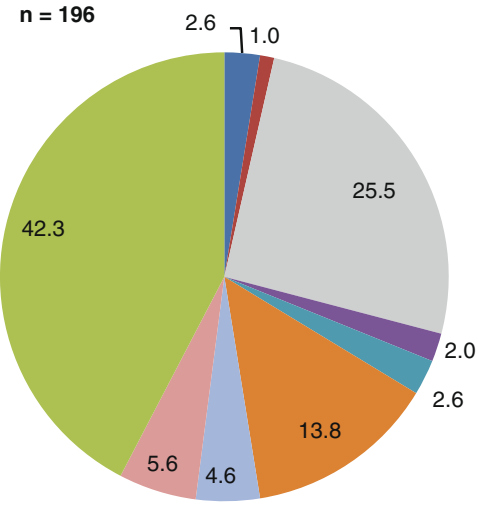

- Other

- Unknown

Alive

Fig. 1 Cause of death among patients enrolled on the registry. Cause of death is shown for a patients aged $<75$ years in LOT1; b patients aged $\geq 75$ years in LOT1; $\mathbf{c}$ patients aged $<75$ years in LOT $\geq 2$; $\mathbf{d}$ patients aged $\geq 75$ years in LOT $\geq 2$. Rounding of values may cause totals to be equal, $>$, or $<100 \%$. CLL chronic lymphocytic leukemia, LOT1 first line of therapy, LOT $\geq 2$ second line of therapy or greater

were similar in both groups (12.8 vs. $13.7 \%$, respectively). In LOT $\geq 2$, febrile neutropenia, thrombocytopenia, and pyrexia were more common in patients $<75$ years (Additional file 4: Table S3 and Additional file 5: Table S4).

\section{Discussion}

While inferior OS is expected in elderly CLL patients, our analysis of elderly CLL patients treated in a 'real-world' setting showed that these patients are more likely to experience CLL- or infection-related deaths. To our knowledge, this has not been reported previously. We developed a prognostic score specifically for this vulnerable patient population, which classified the elderly CLL cohort into high- and lowrisk groups with statistical variation in CLL-related mortality.

As the US population ages, identifying optimal therapeutic strategies for the elderly is a critical unmet medical need as few prospective trials have targeted this patient population. Moreover, elderly patients enrolled in clinical trials might not represent the general elderly population treated in the community. While geriatric assessments should be used to provide an objective and comparable measure of elderly status [20], most studies define elderly patients based solely on an age cut-off. As the median age at diagnosis is 72 years, we selected $\geq 75$ years of age as the cut-off for this analysis. While there are limitations to selecting an age cut-off, we postulated that a cut-off above the median age at diagnosis would be clinically meaningful. In addition, published prospective data on outcomes for patients who are $\geq 75$ years of age are limited [21,22].

Elderly CLL patients were more often treated with rituximab monotherapy than their younger counterparts who were more likely to receive chemoimmunotherapy [22]. However, the fact that $20 \%$ of elderly CLL patients did not receive an anti-CD20 monoclonal antibody is striking, given that all patients were treated after 2010. Even in the younger cohort, we observed that $10 \%$ of patients did not receive any anti-CD20 antibodies. To better understand this variation, we assessed whether patterns of care differed based on 

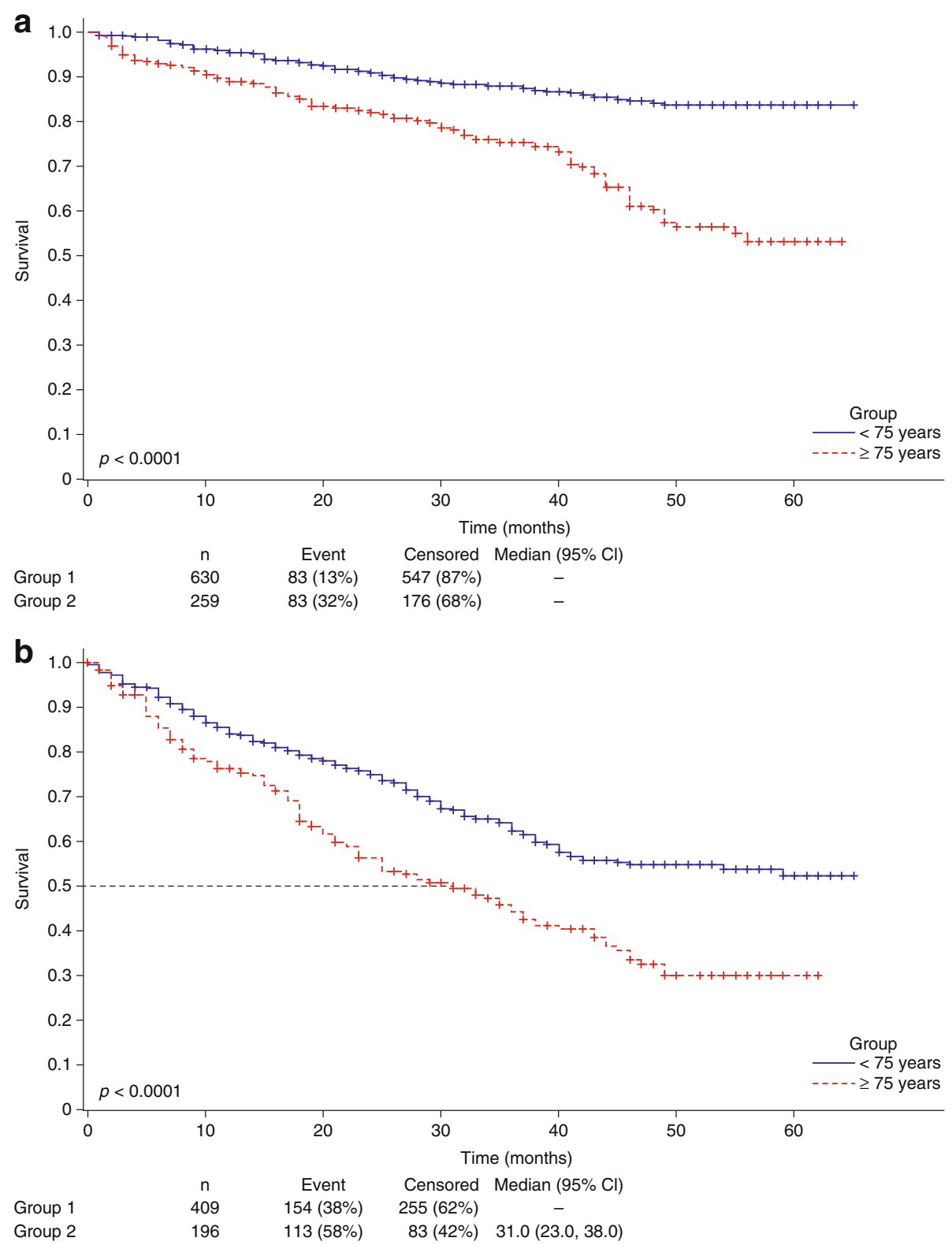

Fig. 2 Overall survival in elderly CLL patients vs. younger patients. Kaplan-Meier curves of OS for patients in a LOT1 and $\mathbf{b}$ LOT $\geq 2$ stratified by age. Percentages are rounded to the nearest whole number. Cl confidence interval, LOT1 first line of therapy, LOT $\geq 2$ second line of therapy or greater, OS overall survival

health insurance coverage or geographic location of the treating institution. Elderly CLL patients were less likely to receive rituximab-based therapies than younger patients, regardless of insurance provider. However, patients residing in the South were more likely to receive anti-CD20 therapy compared with patients living on the West coast. A comparable observation was reported in a study of follicular lymphoma patients in the West of the USA who were less likely to receive rituximab-based maintenance therapy [23]. This may reflect differences in the treating institution and/ or setting. Rituximab use has increased in hospitals while declining in clinics, which could account for the imbalance in treatment between geographic locations [24]. These data suggest that real-world findings differ from clinical trial observations.

Regardless of LOT, responses appeared lower in elderly CLL patients. Although responses were assessed by treating physicians and were not centrally reviewed, CR in the younger patients at LOT1 (42.3\%) was comparable to the response (44\%) reported for treatment-naive patients in the CLL-8 trial of rituximab plus fludarabine/cyclophosphamide [6]. Only $25.9 \%$ of elderly CLL patients achieved a CR in 

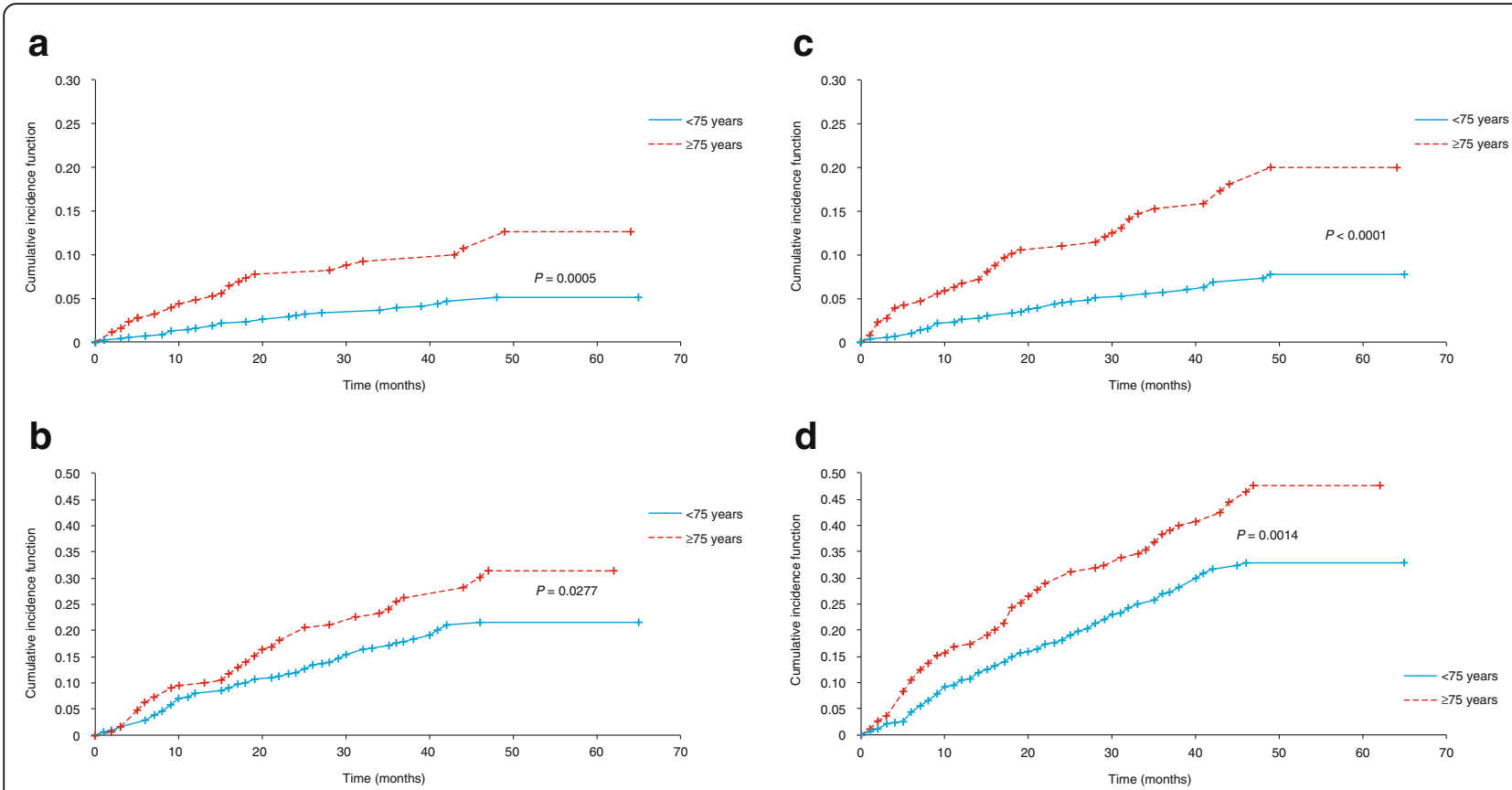

Fig. 3 Cumulative incidence of deaths in elderly CLL patients vs. younger patients. CIF of CLL-related deaths stratified by age in a LOT1 and $\mathbf{b}$ LOT $\geq 2$, and CLL- or infection-related deaths stratified by age in $\mathbf{c}$ LOT1 and $\mathbf{d}$ LOT $\geq 2$, demonstrating increased mortality in elderly CLL patients (red line). Horizontal dashed line shows median survival in patients $\geq 75$ years. Cl confidence interval, CIF cumulative incidence functions, CLL chronic lymphocytic leukemia, LOT1 first line of therapy, LOT $\geq 2$ second line of therapy or greater

LOT1. Given the association between survival and the depth of remission [25], this finding is critical and might contribute to the inferior outcomes noted in our elderly cohort.

Despite the typically indolent nature of CLL, we observed critical outcome differences at a median follow-up of 32.6 months. OS was inferior in elderly CLL patients in any LOT group. Given the predictably inferior OS in the elderly due to competing co-morbidities and deaths from other causes, we compared CLL-related deaths between both groups in LOT1 and LOT $\geq 2$. Only $5 \%$ of patients $<75$ years in LOT1 experienced CLL-specific deaths while $13 \%$ of elderly CLL patients died from CLL alone. This difference was statistically significant $(p=0.0005)$. A similar observation was noted in LOT $\geq 2$ ( $31 \%$ for $\geq 75$ years vs. $22 \%$ for $<75$ years; $p=0.0277$ ). Since infections are a major cause of CLL-related deaths, we evaluated the differences in deaths due to CLL or infection in both LOT groups. The difference remained significant ( $p<0.0001$ in LOT1; $p=0.0014$ in LOT $\geq 2$ ).

We subsequently studied prognostic indicators for CLL- or infection-related deaths in elderly CLL patients. We identified three factors that were significant in a multivariable analysis: time from diagnosis to therapy initiation of $<3$ months, enrollment therapy other than BR, and anemia. While a time from diagnosis to therapy of $<3$ months may suggest patients had more aggressive disease, this is not necessarily related to disease staging. Indeed, the majority of patients in each LOT and age group had Rai stage $0-2$. Rai stage did also not differ significantly between younger and older patients. The prognostic score was used to classify elderly CLL patients according to high- or low-risk of CLL-related death (30.6 vs. $10.3 \%$, respectively; $p=0.0002)$. Contrary to the prognostic models published by Pflug et al. [26], and The International Prognostic Index for patients with CLL (CLL-IPI) working group [27] in which all patients were included regardless of age, our score was specifically designed for elderly CLL patients. Notably, Pflug et al. [26] and the CLL-IPI working group [27] identified older age as an independent factor negatively impacting survival. Our model is also specific to patients receiving therapy as patients under observation were not enrolled to the registry.

Several limitations inherent in any registry-based observational study were encountered during our study. These include the non-random allocation of patients to specific interventions, the assessment of outcomes by non-blinded individuals, and the greater potential for missing data [28]. In the Connect CLL registry, responses were not centrally assessed and indications to treat were based on the treating physician's judgment. Comprehensive molecular and cytogenetic evaluation was missing for some patients. Our analysis also has limitations that are specific to the Connect CLL registry. Only patients requiring therapy were enrolled in the registry. Patients who died without starting therapy were excluded. The registry predates the introduction of BCR-targeted therapies; therefore, the patients in this registry were not treated with these novel agents. As with any registry, patients were treated with a number of 
different therapies. The small size of the cohort and the inclusion of only 181 patients in the prognostic model may also be limiting factors. However, despite the small sample size we believe that these results are meaningful as they relate specifically to elderly patients. Importantly, these data also represent the largest US population of CLL patients treated outside of interventional clinical trials in the chemoimmunotherapy era.

Our finding of increased mortality related to elderly CLL patients highlights the urgent need for therapies tailored to this population and underscores the need to refine CLL treatment for the elderly as current therapies and strategies appear suboptimal. This might reflect a limited enrollment of elderly patients into clinical trials and highlight a flaw in the assumption that effective regimens in younger patients will be effective in elderly patients. As new BCR-targeted agents are increasingly used, their role in elderly CLL patient treatment will require critical analysis to balance efficacy with toxicity. Our data on CLL- and infection-related mortality using traditional therapies are a benchmark against which novel therapies can be measured. Finally, the proposed prognostic score, while requiring validation in patients treated with BCR-targeted therapies, could be used to stratify elderly CLL patients on their enrollment into future clinical trials.

\section{Conclusion}

These data represent the real-world experiences of a large population of CLL patients treated across the USA. Within the limitations of an observational registry we have shown that elderly CLL patients have inferior outcomes with a cumulative increased risk of death from CLL regardless of LOT. Recent improvements in survival for younger patients with CLL have still to be achieved in elderly CLL patients. While elderly people have increased mortality versus younger people regardless of CLL status, it will be important to identify new therapies that can improve the outcomes for elderly CLL patients, similar to the advances seen in younger CLL patients. This unique prognostic model for patients $\geq 75$ years could identify those patients who would benefit from early treatment or treatment with novel therapies.

\section{Additional files}

Additional file 1: List of site-specific IRBs. (DOCX $22 \mathrm{~kb}$ )

Additional file 2: Table S1. CR and ORR of patients $<75$ years versus patients $\geq 75$ years enrolled in LOT1 by specific therapy. (DOCX $17 \mathrm{~kb}$ )

Additional file 3: Table S2. Cause-specific hazards analysis of prognostic indicators for OS. (DOCX $18 \mathrm{~kb}$ )

Additional file 4: Table S3. Incidence of serious adverse events of any grade in enrolled patients by therapy and age group. (DOCX 18 kb)

Additional file 5: Table S4. Incidence of serious adverse events of grade $\geq 3$ in enrolled patients by therapy and age group. (DOCX 18 kb)

\section{Abbreviations}

BCR: B cell receptor; BR: Bendamustine and rituximab; CCl: Charlson comorbidity index; $\mathrm{Cl}$ : Confidence interval; CIF: Cumulative incidence function; CLL: Chronic lymphocytic leukemia; CR: Complete response; E-CLL: Elderly CLL; FCR: Fludarabine, cyclophosphamide, and rituximab; HR: Hazard ratios; IRB: Institutional review board; IWCLL: International Workshop on Chronic Lymphocytic Leukemia; LOT: Line of therapy; ORR: Overall response rate; OS: Overall survival; SAEs: Serious adverse events; SD: Standard deviation

\section{Acknowledgments}

The authors received medical writing support in the preparation of this manuscript from Victoria Edwards, PhD, of Excerpta Medica BV, supported by Celgene Corporation. The Connect ${ }^{\oplus}$ CLL Scientific Steering Committee acknowledges the contributions of all past and current members of the committee for their guidance in the design of the registry and participation in the analysis of the data, including Matthew Davids, Charles Farber, Ian Flinn, Christopher R. Flowers, David L. Grinblatt, Neil E. Kay, Michael Keating, Thomas J. Kipps, Mark F. Kozloff, Nicole Lamanna, Susan Lerner, Anthony Mato, Chadi Nabhan, Chris L. Pashos, Jeff P. Sharman, and Mark Weiss.

\section{Funding}

The Connect CLL registry is sponsored and funded by Celgene Corporation. The sponsor supported the authors in collecting and analyzing the data reported in this registry. The Connect CLL registry was registered at Clinicaltrials.gov on March 4, 2010 as NCT01081015.

\section{Availability of data and materials}

All data generated or analyzed during this study are included in this published article and its additional files.

\section{Author contributions}

CN, AM, CRF, DLG, NL, MAW, MSD and JPS participated in collecting the data. ASS and PK completed the statistical analyses. CN, AM, CRF, DLG, NL, MAW, MSD, ASS, SB, KS, EDF, PK, and JPS participated in interpreting the data reported in this registry. CN, AM, CRF, DLG, NL, MAW, MSD, ASS, SB, KS, EDF, PK, and JPS directed development, review, and approval of this manuscript. All authors are fully responsible for all content and editorial decisions.

\section{Competing interests}

$\mathrm{CN}$ has received research funding from Celgene Corporation, Seattle Genetics, and Genentech, has participated on advisory boards for Celgene Corporation, Astellas, Genentech, and Seattle Genetics, and is currently employed by Cardinal Health.

AM has received research funding from Celgene Corporation, AbbVie, Gilead, Pronai, and TG Therapeutics, has been a consultant for AbbVie, and has been part of a speakers bureau for Celgene Corporation.

CRF has received research funding from Gilead, Spectrum, Millennium, Janssen, Infinity, AbbVie, Acerta, Pharmacyclics, and TG Therapeutics, has been a consultant for Celgene Corporation, Optum Rx, Gilead, Seattle Genetics, Millennium, and Genentech/Roche, and has received honoraria from Celgene Corporation.

DLG has been a consultant and part of a speakers bureau for Celgene Corporation. NL has received research funding from Gilead, AbbVie, Genentech, Infinity, and Pronai, has been a consultant for Gilead, AbbVie, Genentech, Pronai, and Pharmacyclics, and has been on an advisory committee for Celgene Corporation. MAW has been a consultant for Celgene Corporation, Pharmacyclics, and Gilead. MSD has received institutional research funding from, and been on a scientific advisory board for Pharmacyclics, Genentech, Infinity, TG Therapeutics, and has been a consultant for AbbVie, Janssen, Infinity, Celgene Corporation, and Gilead.

PK, ASS, SB, KS, and EDF are employees of Celgene Corporation and have equity. JPS has received research funding from Cilag, Genentech, Gilead, Pharmacyclics, TG Therapeutics, Seattle Genetics, and Acerta, has been a consultant for Cilag, Genentech, Gilead, Pharmacyclics, and Celgene Corporation, and has received honoraria from Genentech and Gilead and travel expenses from Cilag and Gilead. 


\section{Ethics approval and consent to participate}

The study protocol was approved by a central institutional review board (IRB) (Quorum Review IRB, Seattle, WA, USA) or each site's IRB. Prior to patient participation, written informed consent was obtained from each patient (or the patient's legally accepted representative) according to FDA regulatory requirements.

\section{Publisher's Note}

Springer Nature remains neutral with regard to jurisdictional claims in published maps and institutional affiliations.

\begin{abstract}
Author details
${ }^{1}$ Cardinal Health Specialty Solutions, Waukegan, IL 60085, USA. ${ }^{2}$ Center for CLL, Abramson Cancer Center, University of Pennsylvania, Philadelphia, PA 19104, USA. ${ }^{3}$ Emory University, Atlanta, GA 30322, USA. ${ }^{4}$ NorthShore University HealthSystem, Evanston, IL 60201, USA. ${ }^{5}$ Leukemia Service, Hematologic Malignancies Section, Division of Hematology/Oncology, New York-Presbyterian Hospital/Columbia University Medical Center, New York, NY 10032, USA. ${ }^{6}$ Thomas Jefferson University, Philadelphia, PA 19107, USA. ${ }^{7}$ Dana-Farber Cancer Institute, Boston, MA 02215, USA. ${ }^{8}$ Celgene Corporation, Summit, NJ 07901, USA. ${ }^{9}$ Celgene Corporation, Overland Park, KS 66210, USA. ${ }^{10}$ Celgene Corporation, San Francisco, CA, USA. ${ }^{11}$ Willamette Valley Cancer Institute and Research Center, Springfield, OR, USA.
\end{abstract}

Received: 28 September 2016 Accepted: 8 March 2017

Published online: 16 March 2017

\section{References}

1. Siegel RL, Miller KD, Jemal A. Cancer statistics, 2015. CA Cancer J Clin. 2015;65:5-29.

2. Hallek M. Signaling the end of chronic lymphocytic leukemia: new frontline treatment strategies. Blood. 2013;122:3723-34.

3. Catovsky D, Richards S, Matutes E, Oscier D, Dyer MJ, Bezares RF, et al. Assessment of fludarabine plus cyclophosphamide for patients with chronic lymphocytic leukaemia (the LRF CLL4 Trial): a randomised controlled trial. Lancet. 2007:370:230-9.

4. Eichhorst BF, Busch R, Hopfinger G, Pasold R, Hensel M, Steinbrecher C, et al. Fludarabine plus cyclophosphamide versus fludarabine alone in first-line therapy of younger patients with chronic lymphocytic leukemia. Blood. 2006;107:885-91.

5. Flinn IW, Neuberg DS, Grever MR, Dewald GW, Bennett JM, Paietta EM, et al. Phase III trial of fludarabine plus cyclophosphamide compared with fludarabine for patients with previously untreated chronic lymphocytic leukemia: US Intergroup Trial E2997. J Clin Oncol. 2007;25:793-8.

6. Hallek M, Fischer K, Fingerle-Rowson G, Fink AM, Busch R, Mayer J, Hensel $M$, et al. Addition of rituximab to fludarabine and cyclophosphamide in patients with chronic lymphocytic leukaemia: a randomised, open-label, phase 3 trial. Lancet. 2010;376:1164-74.

7. Goede V, Fischer K, Busch R, Engelke A, Eichhorst B, Wendtner CM, et al. Obinutuzumab plus chlorambucil in patients with CLL and coexisting conditions. N Engl J Med. 2014:370:1101-10.

8. Hillmen P, Robak T, Janssens A, Babu KG, Kloczko J, Grosicki S, et al. Chlorambucil plus ofatumumab versus chlorambucil alone in previously untreated patients with chronic lymphocytic leukaemia (COMPLEMENT 1): a randomised, multicentre, open-label phase 3 trial. Lancet. 2015;385:1873-83.

9. Furman RR, Sharman JP, Coutre SE, Cheson BD, Pagel JM, Hillmen P, et al. Idelalisib and rituximab in relapsed chronic lymphocytic leukemia. N Engl J Med. 2014;370:997-1007.

10. Hillmen P, Gribben JG, Follows GA, Milligan D, Sayala HA, Moreton P, et al. Rituximab plus chlorambucil as first-line treatment for chronic lymphocytic leukemia: final analysis of an open-label phase II study. J Clin Oncol. 2014;32:1236-41.

11. Nabhan C, Aschebrook-Kilfoy B, Chiu BC, Smith SM, Shanafelt TD, Evens AM, et al. The impact of race, ethnicity, age, and sex on clinical outcome in chronic lymphocytic leukemia: a comprehensive surveillance, epidemiology, and end results analysis in the modern era. Leuk Lymphoma. 2014:55:2778-84.

12. Brenner H, Gondos A, Pulte D. Trends in long-term survival of patients with chronic lymphocytic leukemia from the 1980s to the early 21 st century. Blood. 2008;111:4916-21.
13. Mato AM, Nabhan C, Kay NE, et al. Real-world clinical experience in the Connect ${ }^{\oplus}$ chronic lymphocytic leukaemia registry: a prospective cohort study of 1494 patients across 199 US centres. Br J Haematol. 2016;175:892-903.

14. Hallek M, Cheson BD, Catovsky D, Caligaris-Cappio F, Dighiero G, Döhner H, et al. Guidelines for the diagnosis and treatment of chronic lymphocytic leukemia: a report from the international workshop on chronic lymphocytic leukemia updating the national cancer institute-working group 1996 guidelines. Blood. 2008;111:5446-56.

15. Charlson ME, Sax FL, MacKenzie CR, Braham RL, Fields SD, Douglas Jr RG. Morbidity during hospitalization: can we predict it? J Chronic Dis. 1978;40:705-12.

16. Charlson ME, Pompei $P$, Ales KL, MacKenzie CR. A new method of classifying prognostic comorbidity in longitudinal studies: development and validation. J Chronic Dis. 1987:40:373-83.

17. Kaplan E, Meier P. Nonparametric estimation from incomplete observations. J Am Stat Assoc. 1958:53:457-81.

18. Kohavi R. A study of cross-validation and bootstrap for accuracy estimation and model selection, vol. 2. Montreal: Proceedings of the 14th International Joint Conference on Artificial Intelligence (IJCAI); 1995. p. 1137-45.

19. Sullivan LM, Massaro JM, D'Agostino Sr RB. Presentation of multivariate data for clinical use: the Framingham study risk score functions. Stat Med. 2004;23:1631-60.

20. Mohile SG, Velarde C, Hurria A, Magnuson A, Lowenstein L, Pandya C, et al. Geriatric assessment-guided care processes for older adults: a Delphi consensus of geriatric oncology experts. J Natl Compr Canc Netw. 2015;13:1120-30.

21. Shanafelt TD, Rabe KG, Kay NE, Zent CS, Jelinek DF, Reinalda MS, et al. Age at diagnosis and the utility of prognostic testing in patients with chronic lymphocytic leukemia. Cancer. 2010;116:4777-87.

22. Satram-Hoang S, Reyes C, Hoang KQ, Momin F, Skettino S. Treatment practice in the elderly patient with chronic lymphocytic leukemia-analysis of the combined SEER and Medicare database. Ann Hematol. 2014;93:1335-44.

23. Nastoupil LJ, Sinha R, Byrtek M, Zhou X, Taylor MD, Friedberg JW, et al. The use and effectiveness of rituximab maintenance in patients with follicular lymphoma diagnosed between 2004 and 2007 in the United States. Cancer. 2014;120:1830-7.

24. DaCosta BS, Small A, Becker LK, Reyes CM. Differences in treatment patterns and health care costs among non-Hodgkin's lymphoma and chronic lymphocytic leukemia patients receiving rituximab in the hospital outpatient setting versus the office/clinic setting. J Cancer Ther. 2014;5:208-16.

25. Fischer K, Bahlo J, Fink AM, Goede V, Herling CD, Cramer $P$, et al. Long-term remissions after FCR chemoimmunotherapy in previously untreated patients with CLL: updated results of the CLL8 trial. Blood. 2016;127:208-15.

26. Pflug N, Bahlo J, Shanafelt TD, Eichhorst BF, Bergmann MA, Elter T, et al. Development of a comprehensive prognostic index for patients with chronic lymphocytic leukemia. Blood. 2014;124:49-62.

27. The International CLL-IPI working group. An international prognostic index for patients with chronic lymphocytic leukemia (CLL-IPI): a meta-analysis of individual patient data. Lancet Oncol. 2016;17:779-90.

28. Levine MN, Julian JA. Registries that show efficacy: good, but not good enough. J Clin Oncol. 2008;26:5316-9.

\section{Submit your next manuscript to BioMed Central and we will help you at every step:}

- We accept pre-submission inquiries

- Our selector tool helps you to find the most relevant journal

- We provide round the clock customer support

- Convenient online submission

- Thorough peer review

- Inclusion in PubMed and all major indexing services

- Maximum visibility for your research

Submit your manuscript at www.biomedcentral.com/submit
) Biomed Central 